\title{
Highly Sensitive Hydrazine Chemical Sensor Based on CNT-PdPt Nanocomposites
}

\author{
Sung Phil Kim, ${ }^{1}$ Seul Gi Lee, ${ }^{1}$ Myong Yong Choi, ${ }^{2}$ and Hyun Chul Choi ${ }^{1}$ \\ ${ }^{1}$ Department of Chemistry, Chonnam National University, Gwangju 500-757, Republic of Korea \\ ${ }^{2}$ Department of Chemistry and Research Institute of Natural Science, Gyeongsang National University, Jinju 660-701, Republic of Korea \\ Correspondence should be addressed to Myong Yong Choi; mychoi@gnu.ac.kr and Hyun Chul Choi; chc12@chonnam.ac.kr
}

Received 24 August 2015; Accepted 1 October 2015

Academic Editor: Shaoming Huang

Copyright (c) 2015 Sung Phil Kim et al. This is an open access article distributed under the Creative Commons Attribution License, which permits unrestricted use, distribution, and reproduction in any medium, provided the original work is properly cited.

\begin{abstract}
Bimetallic PdPt nanoparticles were prepared using the chemical reduction method. The PdPt nanoparticles were successfully deposited on thiolated carbon nanotubes (CNTs) to form a CNT-PdPt nanocomposite as an electron mediator for the fabrication of a hydrazine sensor. The PdPt nanoparticles had an average particle size of $2.3 \mathrm{~nm}$ and were well dispersed on the surfaces of the CNTs in the prepared CNT-PdPt nanocomposite, as demonstrated using transmission electron microscopy (TEM), energy dispersive X-ray spectroscopy (EDX), and X-ray diffraction (XRD). Based on X-ray photoelectron spectroscopy (XPS) results, the estimated proportions of Pd and Pt in the CNT-PdPt nanocomposite were approximately $3.0 \%$ and 3.2\%, respectively. A fabricated chemical sensor based on CNT-PdPt was found to exhibit better amperometric activity with respect to the hydrazine oxidation reaction than CNT-Pd, CNT-Pt, and commercial Pd/C and Pt/C catalysts. This sensor exhibited a linear range of $0.55-1,200 \mu \mathrm{M}$ and a detection limit of $0.28 \mu \mathrm{M}(S / N=3)$ with a fast response time (within $5 \mathrm{~s})$. Furthermore, the sensor could be used repeatedly for the consecutive detection of hydrazine with good reusability and storage stability. These properties demonstrate that the CNT$\mathrm{PdPt}$ nanocomposite is a promising electron mediator for the fabrication of amperometric hydrazine sensors.
\end{abstract}

\section{Introduction}

Hydrazine is a powerful reducing agent and is widely used in industry as a corrosion inhibitor, a pharmaceutical intermediate, a catalyst, an emulsifier, a blowing agent, and rocket fuel $[1,2]$. However, hydrazine is a volatile toxic compound and is readily absorbed via the inhalation and dermal routes. It is also neurotoxic, carcinogenic, mutagenic, and hepatotoxic $[3,4]$. Hydrazine poisoning even at very low concentrations can produce severe problems in living organisms, especially in human beings, such as skin irritation, dermatitis, and damage to the lungs, the liver, and the central nervous system. The recommended maximum level of hydrazine in trade effluents is $1 \mathrm{ppm}$ [5]. Thus, the sensitive and reliable detection of hydrazine is of great analytical importance and has attracted the interest of many researchers. The electrochemical detection of hydrazine is of particular interest because of its high sensitivity, fast response, low cost, simplicity, and suitability for real-time detection. Moreover, the electrochemical oxidation of hydrazine produces nitrogen gas, which is not a pollutant $[6,7]$. However, the direct oxidation of hydrazine on ordinary carbon electrodes is kinetically slow and is accompanied by large overpotentials and small oxidation currents. Therefore, several methods for improving the electron-transfer rate and minimizing the overpotential have been investigated. One promising approach is the use of chemically modified electrodes (CMEs) containing redox mediators immobilized on conventional electrode materials.

Metal nanoparticles and their composites have received a considerable amount of attention due to their large surface areas, good conductivity, and their effective utilization of expensive metals [8-10]. Over the past decade, various metal nanoparticles such as Pt [11], Pd [12-14], Au [15, 16], and Ag [17] have been used to fabricate CMEs for the electrochemical oxidation of hydrazine. Previous studies have demonstrated that the electrochemical activities of nanoparticles are strongly dependent on their composition [18], size [19], and degree of dispersion on CMEs [20]. Thus, the development of high-performance catalysts with high activity, stability, and recyclability is crucial. Recently, bimetallic nanoparticles 


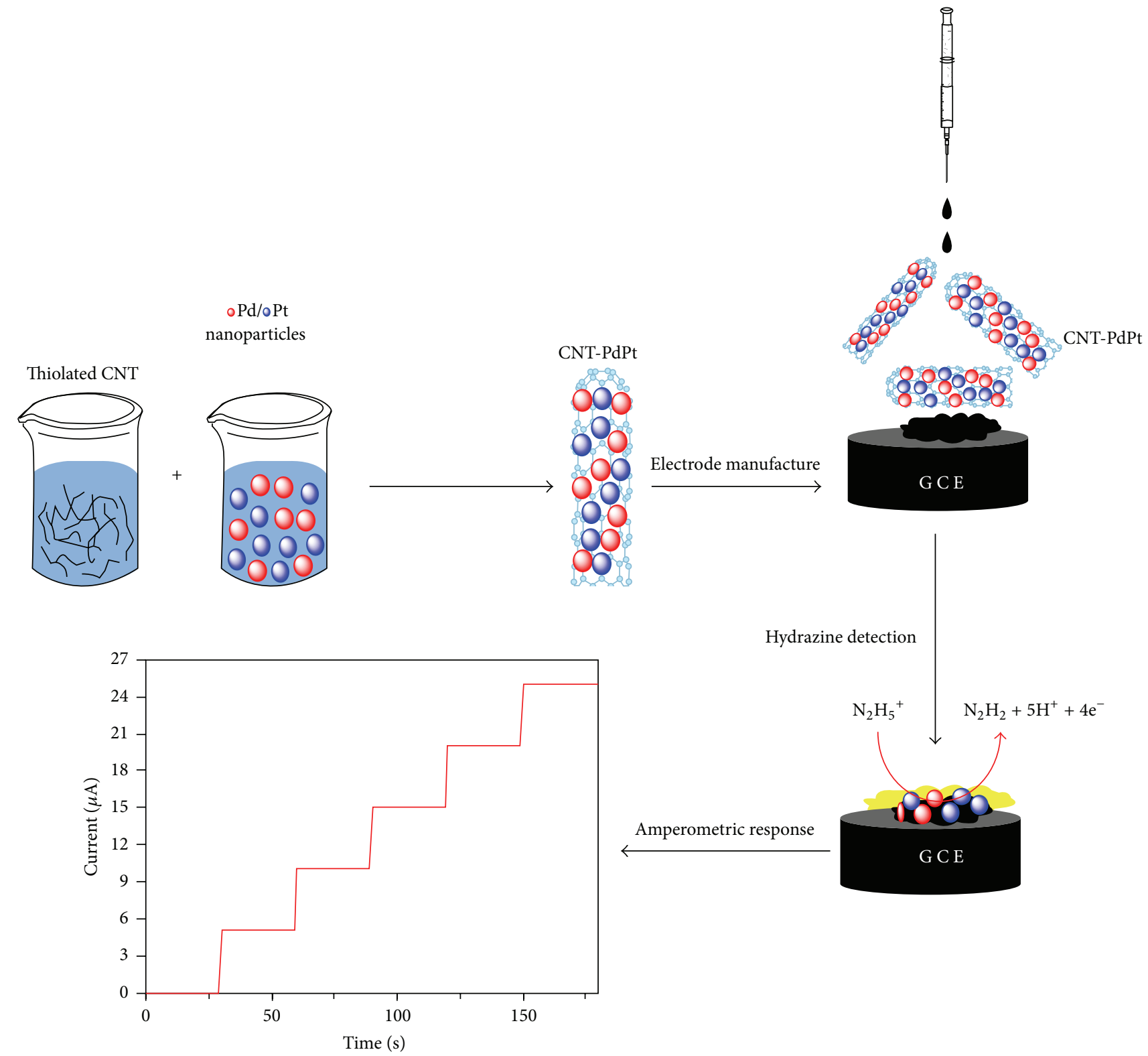

SCHEME 1: Schematic illustration of the fabricated amperometric hydrazine sensor based on CNT-PdPt modified electrode.

have drawn considerable attention because of their unique electronic, optical, and catalytic properties. It is known that bimetallic nanoparticles can exhibit synergetic catalytic improvement effects not found in their monometallic counterparts, which result in higher efficiencies in various catalytic processes such as chemical sensor, hydrogen generation from hydrogen storage materials, hydrogenation, fuel cell catalytic reforming, and electrochemical alcohol oxidation[21]. However, only a few studies have examined the use of bimetallic nanoparticles in hydrazine sensing.

Herein, we report the synthesis of bimetallic PdPt nanoparticles uniformly deposited on thiolated carbon nanotubes (CNTs). Thiol groups were utilized as linkers to secure the PdPt nanoparticles without agglomeration. The structural and surface properties of the prepared CNT-PdPt nanocomposites were characterized in detail, and then the nanocomposites were utilized as electron mediators for the fabrication of highly sensitive hydrazine chemical sensors (see Scheme 1). The as-prepared CNT-PdPt nanocomposite modified glassy carbon (GC) electrode was found to exhibit a linear response to hydrazine in the range from 0.55 to $1,200 \mu \mathrm{M}$ with the detection of $0.28 \mu \mathrm{M}$. Moreover, the modified electrode has good anti-interference ability, reusability, and long-term storage stability.

\section{Experimental}

2.1. Chemicals. Multiwalled carbon nanotubes were obtained from Carbon Nano Tech. Co., Ltd. (South Korea). Sodium hydrogen sulfide $(\mathrm{NaSH})$, chloroplatinic acid hexahydrate $\left(\mathrm{H}_{2} \mathrm{PtCl}_{6} \cdot 6 \mathrm{H}_{2} \mathrm{O}\right)$, sodium tetrachloropalladate $\left(\mathrm{Na}_{2} \mathrm{PdCl}_{4}\right)$, and Nafion (5 wt.\% in a mixture of lower aliphatic alcohols 
and water) were purchased from Sigma-Aldrich. All other chemicals were of analytical-reagent grade and used without further purification. All aqueous solutions were prepared with doubly distilled water by using a Direct-Q 3 water purification system (Millipore Co. Ltd., USA).

2.2. Preparation of Thiolated CNTs. The CNTs were stirred in an acid solution of $\mathrm{HNO}_{3}$ and $\mathrm{H}_{2} \mathrm{SO}_{4}(1: 3$ by volume $)$ at $90^{\circ} \mathrm{C}$ for $3 \mathrm{~h}$, then filtered, washed with distilled water, and dried in an oven at $110^{\circ} \mathrm{C}$. The acid-treated CNTs were dispersed in tetrahydrofuran (THF) and then $\mathrm{NaSH}$ aqueous solution was added to produce thiol groups on the CNT surfaces. Thiolation was confirmed by examining the XPS spectrum in the sulfur $2 \mathrm{p}$ region. The thiolated CNTs were filtered, washed with distilled water, and dried under vacuum at $80^{\circ} \mathrm{C}$ for $4 \mathrm{~h}$.

2.3. Preparation of CNT-PdPt. Firstly, aqueous solutions of $\mathrm{H}_{2} \mathrm{PtCl}_{6} \cdot 6 \mathrm{H}_{2} \mathrm{O}(0.4 \mathrm{~g} / 100 \mathrm{~mL})$ and $\mathrm{Na}_{2} \mathrm{PdCl}_{4}(0.4 \mathrm{~g} / 100 \mathrm{~mL})$ were prepared and then stirred for $30 \mathrm{~min}$. A freshly prepared $0.1 \mathrm{M} \mathrm{NaBH}_{4}$ solution was added drop-wise (metal: $\mathrm{NaBH}_{4}$ $=1: 1 \mathrm{w} / \mathrm{w}$ ) under continuous vigorous stirring, which produced a black colloidal dispersion. In the preparation of a CNT-PdPt catalyst, a thiolated CNT sample $(300 \mathrm{mg})$ was dispersed with $20 \mathrm{~min}$ ultrasonic vibration into $50 \mathrm{~mL}$ of anhydrous ethanol. The prepared colloidal solution $(80 \mathrm{~mL})$ was then added to the CNT solution under $10 \mathrm{~h}$ stirring at $55^{\circ} \mathrm{C}$. The CNT-PdPt catalysts were finally obtained after centrifugation, washing with absolute ethanol, and vacuum drying at $40^{\circ} \mathrm{C}$ overnight.

2.4. Physicochemical Characterization. Transmission electron microscopy (TEM) images were obtained with a TecnaiF20 system operated at $200 \mathrm{kV}$. The samples for TEM analysis were manufactured on a carbon-coated $\mathrm{Cu}$ grid by dipcoating in dilute solutions ( $\sim 1.0 \mathrm{wt} . \%$ solid content). The diameters of the attached PdPt nanoparticles were measured by using iTEM software (Soft Imaging System GmbH), counting 400 particles. For nonsymmetrical particle shapes, both the largest and shortest distances were measured to acquire an average value for the particle diameter. X-ray diffraction (XRD) analysis was performed with an X'Pert Pro high-resolution X-ray diffractometer (PANalytical, Netherlands) utilizing $\mathrm{Cu} \mathrm{K} \alpha$ radiation $(\lambda=1.5406 \AA)$ as the $\mathrm{X}$ ray source. Data were collected at room temperature in the range from $20^{\circ}$ to $75^{\circ}$ at increments of $0.05^{\circ}$. Raman spectra were obtained at room temperature by using an inVia Reflex (Renishaw 1000) micro-Raman spectrometer with a $514.5 \mathrm{~nm}$ laser line. Auger electron spectroscopy (AES) was carried out by using a PHI 700 Scanning Auger Nanoprobe with an incident electron energy of $10 \mathrm{keV}$. The focused beam diameter was approximately $1.0 \mu \mathrm{m}$. X-ray photoelectron spectroscopy (XPS) analysis was performed with a VG Multilab 2000 spectrometer (Thermo VG Scientific) in an ultrahigh vacuum. This system employs an unmonochromatized $\mathrm{Mg}$ $\mathrm{K} \alpha(1253.6 \mathrm{eV})$ source and a spherical section analyzer.

2.5. Electrochemical Characterization. A GC electrode with a diameter of $3.0 \mathrm{~mm}$ was used as the substrate to confine the prepared CNT-PdPt catalysts for electrochemical investigation. Prior to the surface coating, the GC electrode was highly polished with alumina paste and rinsed several times with distilled water before a final rinse in methanol. $3.0 \mu \mathrm{L}$ of the sample suspension in DI water $(1.0 \mathrm{~g} / \mathrm{L})$ was then dropped on the surface of the pretreated GC. Finally, the CNT-PdPt-coated GC electrode was covered again with 5\% Nafion solution. A small amount of Nafion is commonly used to prepare a net-like film that prevents deposited materials on electrodes from dissolution. The thickness of the Nafion film was calculated to be $0.6 \mu \mathrm{m}$ from the known weight of the Nafion solution and the density $\left(1.58 \mathrm{~g} / \mathrm{cm}^{3}\right)$ of wet Nafion films [22]. For comparison, bare GC, CNT-Pd, CNT$\mathrm{Pt}$, and commercial $\mathrm{Pd} / \mathrm{C}$ and $\mathrm{Pt} / \mathrm{C}$ were fabricated with the method described above. The electrochemical measurements were performed by using a potentiostat (EC epsilon potentiostat/galvanostat). All experiments were carried out by using a conventional three-electrode system with a GC electrode or a modified electrode as the working electrode, a saturated calomel electrode (SCE), and a platinum wire as the counter electrode. A $0.1 \mathrm{M}$ phosphate buffer solution (PBS, pH 7.4) was used as the supporting electrolyte. The electrolyte was bubbled with Ar gas for at least 30 min to remove oxygen. Amperometric measurements were carried out under stirred conditions.

\section{Results and Discussion}

3.1. Morphological and Structural Characterizations of CNTPdPt Nanocomposites. Figure 1(a) shows a typical TEM image of a CNT-PdPt nanocomposite, which confirms the high dispersion of the nanosized particles on the CNTs; smaller and highly dispersed nanoparticles are much more abundant than larger, aggregated nanoparticles. Most of the nanoparticles in the CNT-PdPt nanocomposites range in size from 2 to $4 \mathrm{~nm}$. The adhered nanoparticles could not be separated from the CNTs, even with thorough washing and prolonged sonication. The corresponding EDX spectrum confirms the presence in the nanoparticles of both $\mathrm{Pd}$ and Pt with an atomic composition of $49 \% \mathrm{Pd}$ and $51 \% \mathrm{Pt}$, and thus bimetallic PdPt nanoparticles are present (Figure 1(a), inset). Figure 1(b) shows a particle-size histogram of a CNT-PdPt nanocomposite and its fit with a Gaussian-shape curve. The nanoparticles have an average diameter of $2.3 \pm$ $0.03 \mathrm{~nm}$ within a narrow distribution. The crystal structure of the nanoparticles was investigated with XRD, as shown in Figure 1(c). The diffraction peak around $25^{\circ}$ arises from the (002) reflection of the graphite layers in the CNTs [23]. The peaks near $39.8^{\circ}, 45.9^{\circ}$, and $67.4^{\circ}$ are associated with the (111), (200), and (220) planes, respectively, of the face-centered cubic structure of Pd and Pt. These XRD results show that metal nanoparticles have successfully been prepared. It is however impossible to distinguish between Pd and Pt in the XRD pattern because the lattice constants of these metals are very close (the lattice mismatch is $0.77 \%$ ) [24, 25]. In addition, the width of the XRD peaks can provide information on the average size of nanoparticles. According to Scherrer equation, the average crystalline size of the PdPt grains on 


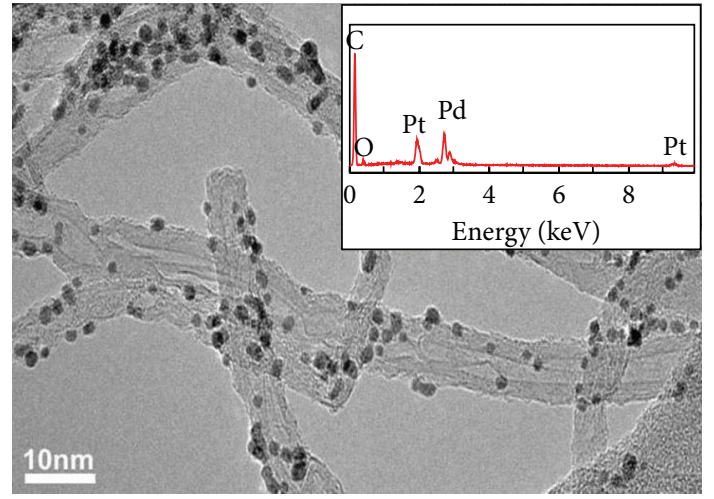

(a)

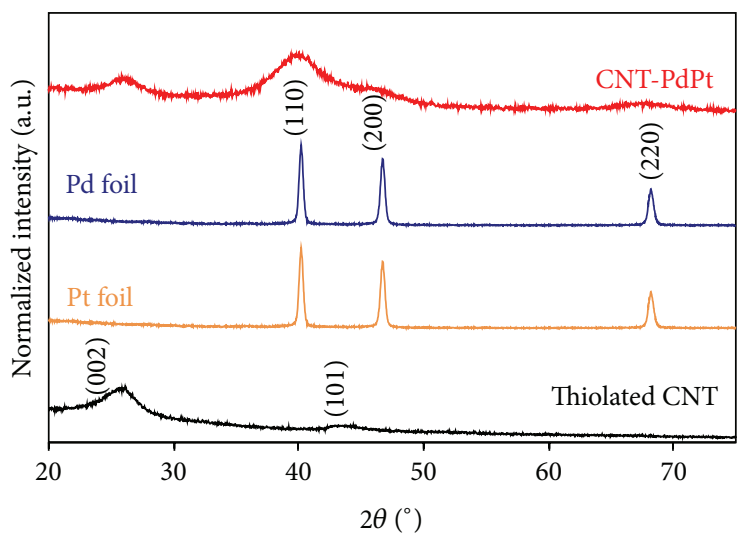

(c)

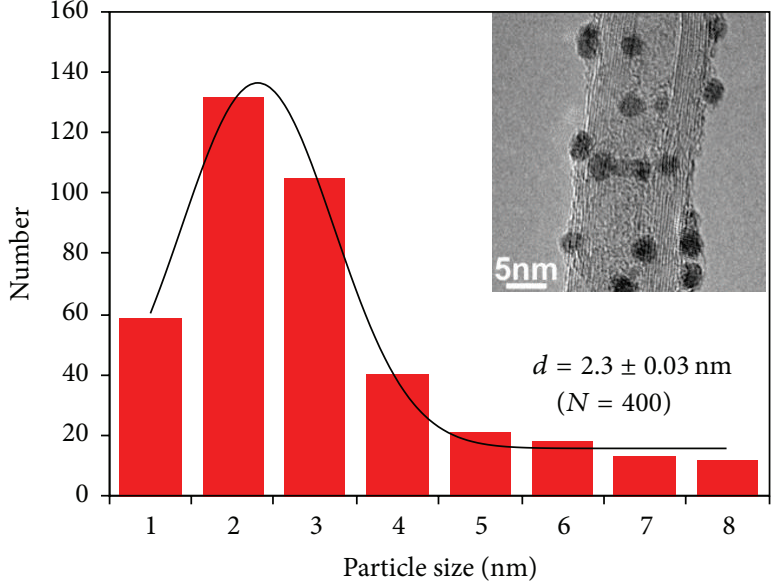

(b)

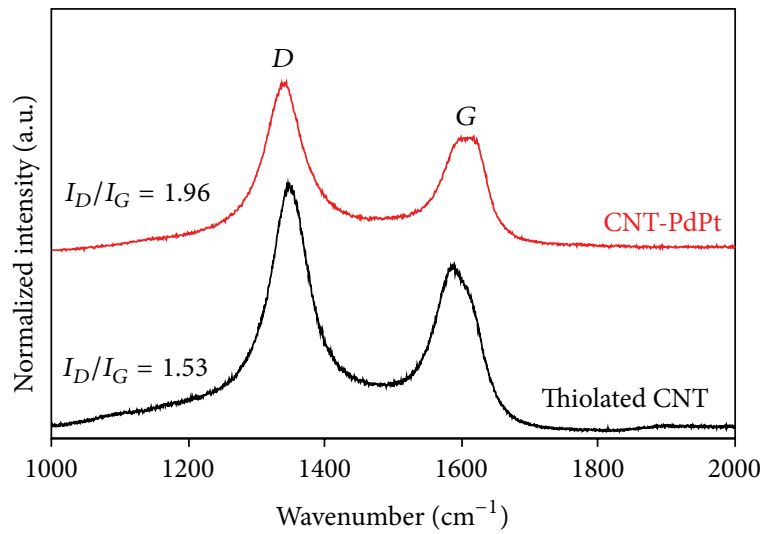

(d)

FIGURE 1: (a) TEM image of CNT-PdPt, with corresponding EDX spectrum (inset). (b) Size distribution histogram of nanoparticles in CNTPdPt. The solid line in the histogram graph represents a Gaussian fitting curve. Inset is the high resolution image of deposited nanoparticles. (c) XRD patterns of thiolated CNT, Pd foil, Pt foil, and CNT-PdPt. (d) Room temperature Raman spectra of thiolated CNT and CNT-PdPt obtained with laser excitation at $514.5 \mathrm{~nm}$, where $I_{D}$ and $I_{G}$ denote $D$ and $G$ band intensities, respectively.

the CNT surface is calculated to be $3.2 \mathrm{~nm}$. The TEM and XRD results confirm that PdPt nanoparticles were anchored to the surfaces of the thiolated CNTs. Raman spectroscopy was used to characterize the crystallinities of the CNT supports before and after metal deposition. As shown in Figure 1(d), the spectrum consists of two bands at $\sim 1330 \mathrm{~cm}^{-1}$ ( $D$ band) and $\sim 1570 \mathrm{~cm}^{-1}$ ( $G$ band). The $D$ band is a disorderinduced feature originating from the vibrations of $C$ atoms with dangling bonds, and the $G$ band arises from a tangential shear mode of the $\mathrm{C}$ atoms that corresponds to the stretching mode in the graphite plane $[26,27]$. The $D / G$ band intensity ratio $\left(I_{D} / I_{G}\right)$ is in linear relation to the inverse of the in-plane crystallite dimension. The $I_{D} / I_{G}$ value is approximately 1.53 for the thiolated CNTs and approximately 1.96 for CNT-PdPt. These results indicate that the deposition of the nanoparticles onto the CNTs results in a decrease in their crystallinity.

Figure 2 shows Auger spectroscopic data for CNT-PdPt. $\mathrm{C}, \mathrm{S}, \mathrm{O}, \mathrm{Pt}$, and $\mathrm{Pd}$ peaks are evident in the spectrum around $280,135,520,67$, and $335 \mathrm{eV}$, respectively. The relative surface atomic ratio was estimated from the corresponding peak areas, corrected with tabulated sensitivity factors [28].

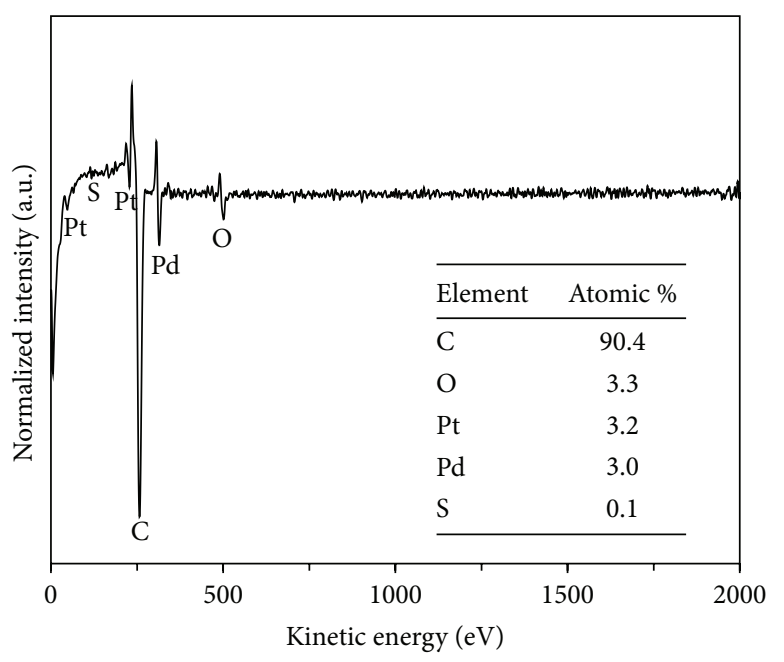

Figure 2: Auger spectrum of CNT-PdPt.

The estimated proportions of Pd and Pt in the samples are nearly 3.0 and $3.2 \%$, respectively. The oxidation states of 


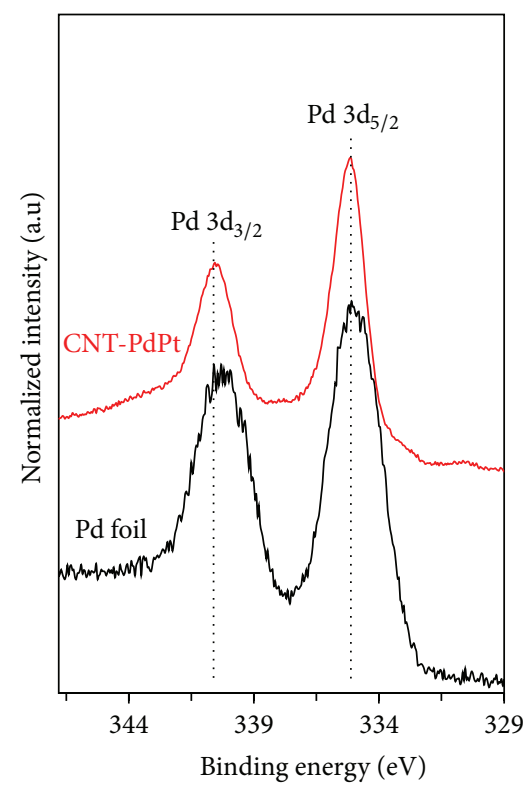

(a)

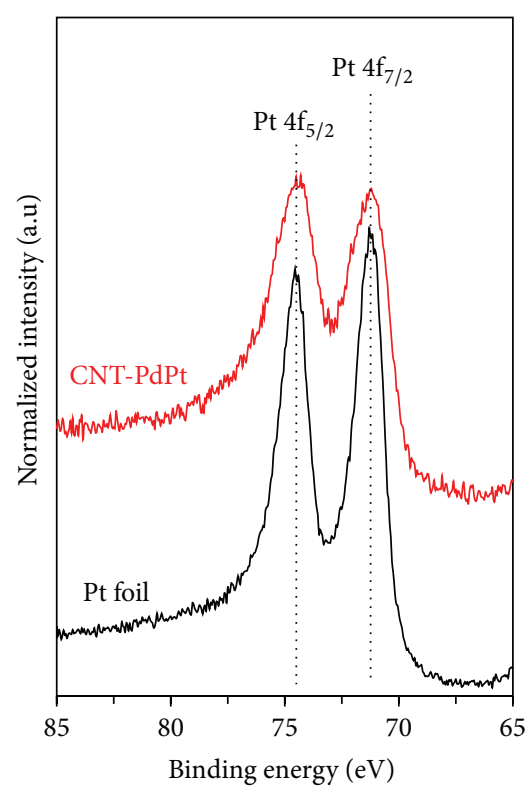

(b)

FIgUre 3: (a) Pd 3d core level XPS spectra of CNT-PdPt. (b) Pt 4f core level XPS spectra of CNT-PdPt.

the Pd and Pt species deposited on the CNTs were verified with XPS measurements. Figure 3(a) shows the Pd 3d XPS spectrum for CNT-PdPt; the two peaks centered at $335.1 \mathrm{eV}$ and $340.8 \mathrm{eV}$ are the $P d 3 d_{5 / 2}$ and $3 d_{3 / 2}$ photoelectron lines, respectively. Figure $3(\mathrm{~b})$ shows the Pt $4 \mathrm{f}$ XPS spectrum; the Pt $4 \mathrm{f}_{7 / 2}$ and $\mathrm{Pt} 4 \mathrm{f}_{5 / 2}$ lines appear at $71.2 \mathrm{eV}$ and $74.5 \mathrm{eV}$, respectively. These values are consistent with the binding energies of the reference Pd and Pd foils.

\subsection{Electrochemical Behavior of CNT-PdPt Nanocomposites.} The electrocatalytic activity of the CNT-PdPt nanocomposites with respect to the direct electrooxidation of hydrazine was examined by performing cyclic voltammetry in $0.1 \mathrm{M}$ PBS (pH 7.4). Typical results obtained with a potential scan from -0.4 to $0.8 \mathrm{~V}$ versus SCE are shown in Figure 4. No redox peaks have been observed in the absence of hydrazine indicating that the modified electrode is nonelectroactive in the selected potential region. However, after injecting $50 \mathrm{mM}$ hydrazine into the PBS, a remarkable oxidation peak at about $0.22 \mathrm{~V}$ is observed. This result indicates that the observed current originates from hydrazine oxidation. For comparison, the cyclic voltammogram of thiolated CNTs is also presented (Figure 4, inset). No obvious oxidation current arises for the thiolated CNTs over most of the potential range. Thus the observed current in the CNT-PdPt case arises from the oxidation of chemisorbed hydrazine on the surface of the PdPt nanoparticles.

The overall reaction of hydrazine is known to be a fourelectron process [29-31]. To confirm the electrochemical mechanism, the cyclic voltammograms for $3 \mathrm{mM}$ hydrazine in $0.1 \mathrm{M} \mathrm{PBS}$ ( $\mathrm{pH} 7.4$ ) were recorded at different scan rates $(v)$. The obtained results are shown in Figure 5(a). The oxidation peak current $\left(I_{p}\right)$ gradually increases and the oxidation peak potential $\left(E_{p}\right)$ shifts to more positive values with increasing scan rates. As shown in Figure 5(b), a plot

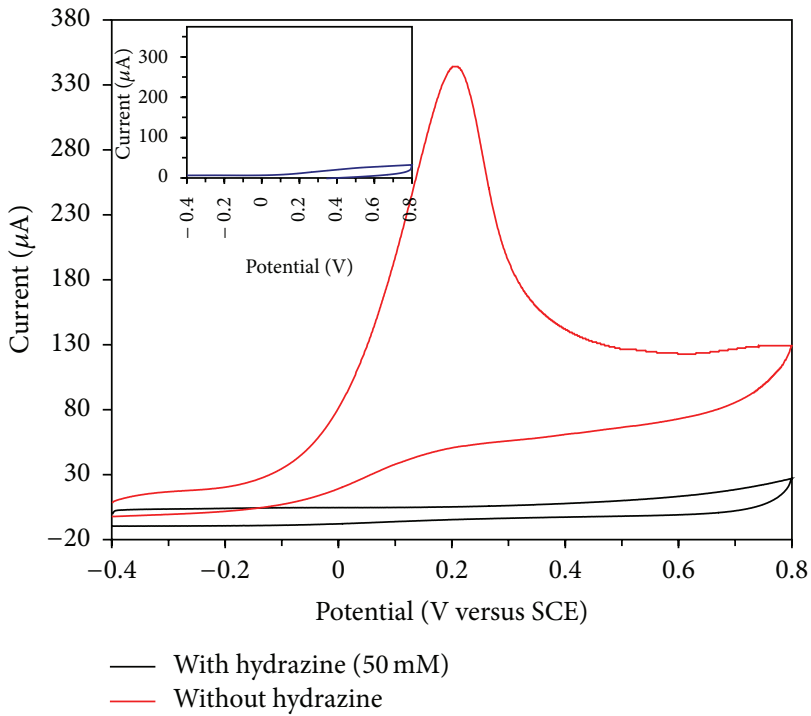

Figure 4: Cyclic voltammograms for CNT-PdPt in the absence and presence of $50 \mathrm{mM}$ hydrazine. The insert is the cyclic voltammogram for thiolated CNT electrode in the presence of $50 \mathrm{mM}$ hydrazine. Scan rate: $50 \mathrm{mV} / \mathrm{s}$.

of $I_{p}$ versus $v^{1 / 2}$ exhibited a linear relationship, indicating that the oxidation process is controlled by diffusion. The corresponding linear regression equation can be expressed as $I_{p}(\mu \mathrm{A})=1.604 v^{1 / 2}+1.145, R^{2}=0.998$. In addition, a linear relationship between $E_{p}$ and $\log (v)$ was obtained as shown in Figure 5(c). According to Tafel equation, the relationship of $E_{p}$ with $\log (v)$ was expressed as the following equation:

$$
E_{p}=\left(\frac{b}{2}\right) \log (v)+k
$$




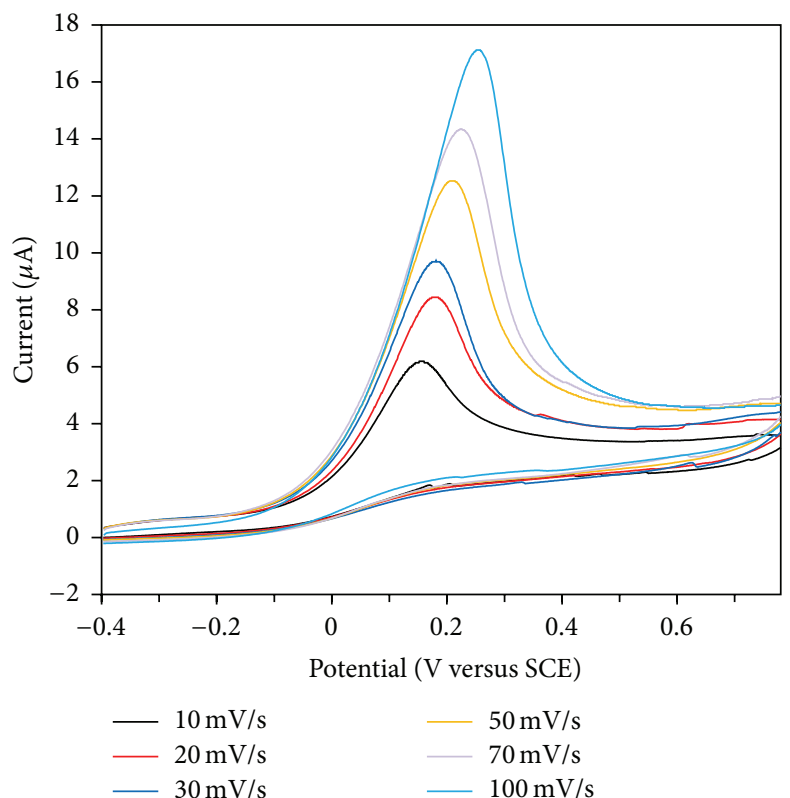

(a)

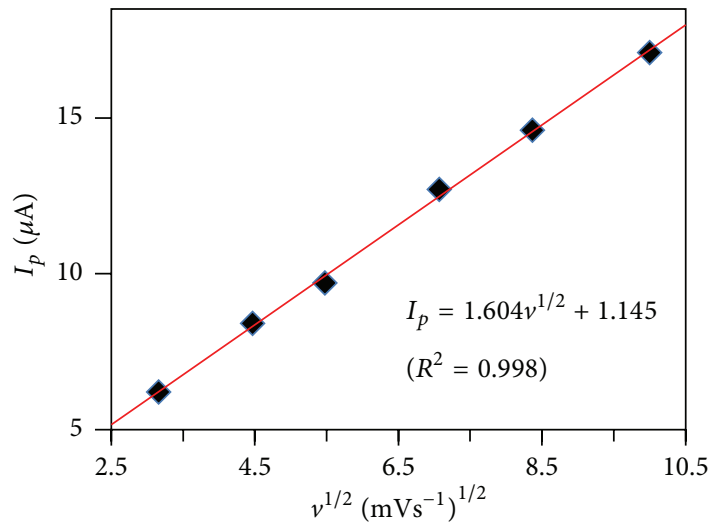

(b)

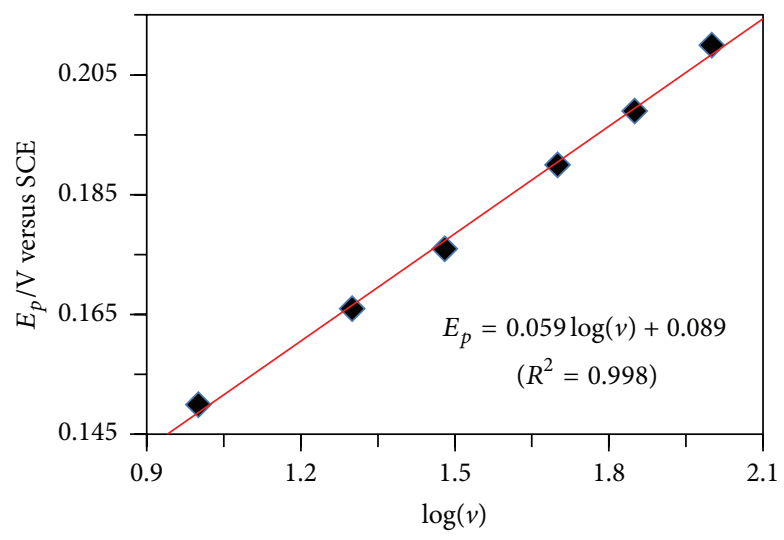

(c)

Figure 5: (a) Cyclic voltammograms for CNT-PdPt in the presence of $3 \mathrm{mM}$ hydrazine in $0.1 \mathrm{M}$ PBS (pH 7.4) at different scan rates: 10, 20, 30, 50,70 , and $100 \mathrm{mVs}^{-1}$. (b) Plot of peak current versus square root of scan rate. (c) Plot of oxidation peak potential versus logarithm of scan rate.

where $b$ is the Tafel slope and $k$ is a constant value. The plot of $E_{p}$ versus $\log (v)$ shows that the slope is $0.059 \mathrm{~V}$, and thus $b=0.118 \mathrm{~V}$. The Tafel slope can also be obtained from the following equation: $b=0.059 /(1-\alpha) n_{a}(\alpha$, the transfer coefficient; $n_{a}$, the number of electrons involved in the rate-determining step) [32]. Supposing one electron is transferred in the rate-determining step $\left(n_{a}=1\right)$, the value of $\alpha$ for the hydrazine oxidation process is estimated to be 0.50 . The number of total electrons $(n)$ involved in the overall hydrazine oxidation process can be obtained from the slope of $I_{p}$ versus $v^{1 / 2}$ according to the following equation for a totally irreversible diffusive process [33]:

$$
I_{p}=3.01 \times 10^{5} n\left[(1-\alpha) n_{a}\right]^{1 / 2} A C_{s} D^{1 / 2} v^{1 / 2}
$$

where $A, C_{s}$, and $D$ are electrode surface area, hydrazine concentration, and diffusion coefficient of hydrazine, respectively. By considering $\left[(1-\alpha) n_{a}\right]=0.50$ and $D=$ $5.03 \times 10^{-6} \mathrm{~cm}^{2} \mathrm{~s}^{-1}$ [28], the total number $(n)$ of electrons involved in the oxidation of hydrazine is calculated to be 4 . Thus, the oxidation of hydrazine on CNT-PdPt occurs via the following reaction:

$$
\mathrm{N}_{2} \mathrm{H}_{5}^{+} \longrightarrow \mathrm{N}_{2}+5 \mathrm{H}^{+}+4 \mathrm{e}^{-}
$$

Note that hydrazine has $\mathrm{pK}_{\mathrm{a}}$ of 8.1 at $25^{\circ} \mathrm{C}$ and that the most stable form of hydrazine in a $\mathrm{pH} 7.4$ solution is its protonated state (hydrazinium, $\mathrm{N}_{2} \mathrm{H}_{5}{ }^{+}$) [19].

\subsection{Hydrazine Chemical Sensor of CNT-PdPt Nanocomposites.} Figure 6 displays a typical amperometric plot for CNTPdPt in $0.1 \mathrm{M}$ PBS upon the successive addition of $1.0 \mu \mathrm{M}$ hydrazine at an applied potential of $0.2 \mathrm{~V}$ under convection provided by a magnetic stirrer operating at constant speed. 
TABLE 1: Comparisons of the responses of some hydrazine sensors constructed by different nanoparticles-based mediators.

\begin{tabular}{|c|c|c|c|}
\hline Electrode material & Detection limit $(\mu \mathrm{M})$ & Linear dynamic range $(\mu \mathrm{M})$ & Reference \\
\hline Pd-AuNPs/GCE ${ }^{\mathrm{a}}$ & 0.07 & $0.1-500$ & {$[14]$} \\
\hline $\mathrm{PdNPs} / \mathrm{rGO} / \mathrm{RDE}^{\mathrm{b}}$ & 0.20 & $10-300$ & [19] \\
\hline Pd nanoparticles-EDAC $/ \mathrm{GCE}$ & 1.5 & $5-150$ & {$[30]$} \\
\hline Polyaniline $/ \mathrm{Au}_{0} / \mathrm{GCE}$ & 1.0 & $10-6,000$ & {$[31]$} \\
\hline AuNPs & 1.1 & $6-36$ & {$[34]$} \\
\hline Pd-MWNT & 1.0 & $10-70$ & [35] \\
\hline $\mathrm{AuCu}-\mathrm{EGN}-\mathrm{IL}^{\mathrm{e}}$ & 0.10 & $0.2-110$ & [36] \\
\hline AuNPs/rGO/GCE & 0.08 & $5-900$ & {$[37]$} \\
\hline Polyaniline/Pd & 0.05 & $350-12,000$ & {$[38]$} \\
\hline CNT-PdPt & 0.28 & $0.55-1,200$ & This work \\
\hline
\end{tabular}

${ }^{a}$ Glassy carbon electrode.

${ }^{\mathrm{b}}$ Rotating disk electrodes.

${ }^{c}$ Ethylenediamine cellulose.

${ }^{\mathrm{d}}$ Multiwalled carbon nanotube.

${ }^{\mathrm{e}}$ Electrodeposited on a graphene-ionic liquid.

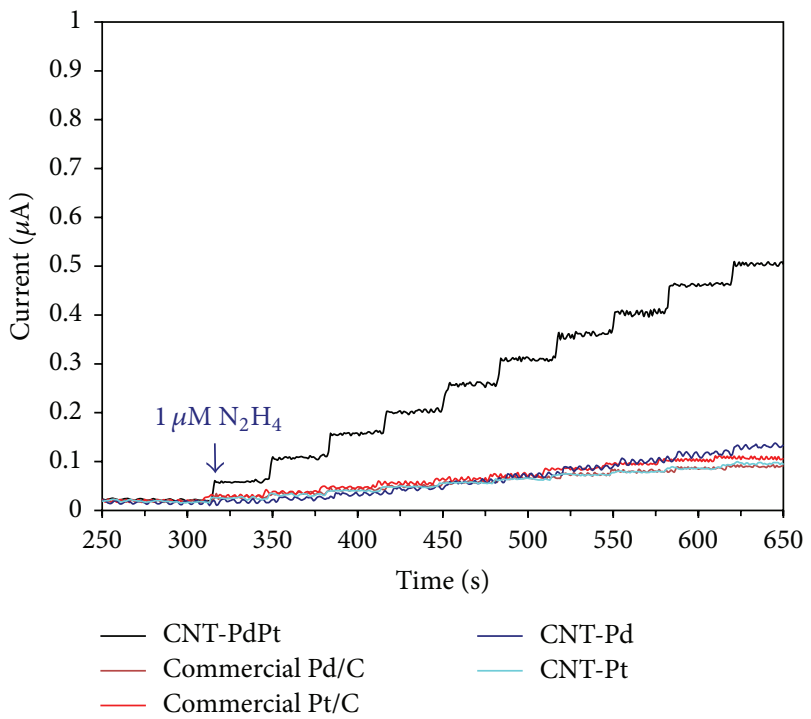

FIGURE 6: Amperometric response of the CNT-PdPt held at $0.2 \mathrm{~V}$ in $0.1 \mathrm{M}$ PBS ( $\mathrm{pH}$ 7.4) for successive additions of $1.0 \mu \mathrm{M}$ hydrazine. For comparison, the amperometric responses of CNT-Pd, CNT-Pt, and commercial $\mathrm{Pd} / \mathrm{C}$ and $\mathrm{Pt} / \mathrm{C}$ catalysts are also shown.

For comparison, the amperometric responses of CNT-Pd, $\mathrm{CNT}-\mathrm{Pt}$, and commercial $\mathrm{Pd} / \mathrm{C}$ and $\mathrm{Pt} / \mathrm{C}$ catalysts are also displayed. In the beginning, the amperometric experiment is performed in PBS without hydrazine for stabilizing the background current. Successive addition of $1.0 \mu \mathrm{M}$ hydrazine to a stirred solution resulted in stepwise increase of anodic current, but the amplitudes of increase of different electrodes differed dramatically. It can be seen that the CNTPdPt nanocomposites display much higher current response than that of other electrodes, indicating that the CNTPdPt nanocomposite is a highly effective catalyst for the detection of hydrazine at low concentrations. The enhanced current responses of CNT-PdPt can be attributed to the superior mass transport and the large effective surface area resulting from the smaller size and excellent dispersion of PdPt nanoparticles on the CNT.

In order to estimate the linear response range and the detection limit, amperometric curves were recorded for CNT-PdPt at various concentrations of hydrazine, as shown in Figure 7(a). When an aliquot of hydrazine was dropped into the stirred PBS solution, the amperometric responses achieved steady state within 5 seconds, which is fast amperometric behavior. The magnitudes of the response currents are dependent on the hydrazine concentration. Figure $7(\mathrm{~b})$ shows a plot of the variation of the response current with the hydrazine concentration. The response current increases with increasing hydrazine concentration and is linear in the concentration range of $0.55-1,200 \mu \mathrm{M}$ with the linear equation $i(\mu \mathrm{A})=0.0424 C(\mu \mathrm{M})+0.2140\left(R^{2}=0.9981\right)$. The detection limit can be determined by the following equation: $\mathrm{LOD}=3 S_{b} / \mathrm{m}$, where LOD is the detection limit, $S_{b}$ $(0.0040 \mu \mathrm{A})$ is the standard deviation of the blank response, and $\mathrm{m}(0.0424 \mu \mathrm{A} / \mu \mathrm{M})$ is the slope of the calibration curve. The detection limit was found to be $0.28 \mu \mathrm{M}(S / N=3)$. A comparison of the results of the present study with those of some other studies of hydrazine oxidation is given in Table 1. As can be seen in this table, the detection limit and linear range are comparable to or better than previous results for hydrazine determination with various metal nanoparticlebased electrodes.

Anti-interference properties, reproducibility, and reusability are additional important criteria for the success of sensing applications. Possible interferences for the determination of hydrazine at the CNT-PdPt electrode were investigated by adding various foreign species into the hydrazine solution; the results are shown in Figure 8(a). No clear interference was found for the CNT-PdPt nanocomposite upon the addition of interfering species, such as 8-fold quantities of $\mathrm{NH}_{3}, \mathrm{NO}_{2}{ }^{-}, \mathrm{NO}_{3}{ }^{-}, \mathrm{Na}^{+}, \mathrm{Ca}^{2+}, \mathrm{Cl}^{-}, \mathrm{SO}_{4}{ }^{2-}$, ethanol, and glucose. The electrode was found to display strong current responses to subsequent hydrazine additions, which demonstrates that CNT-PdPt possesses a high 


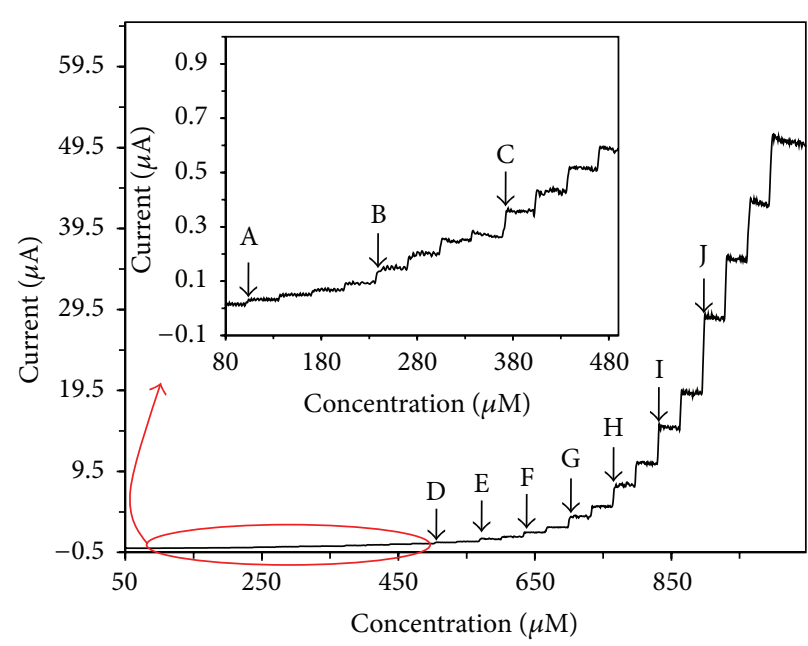

(a)

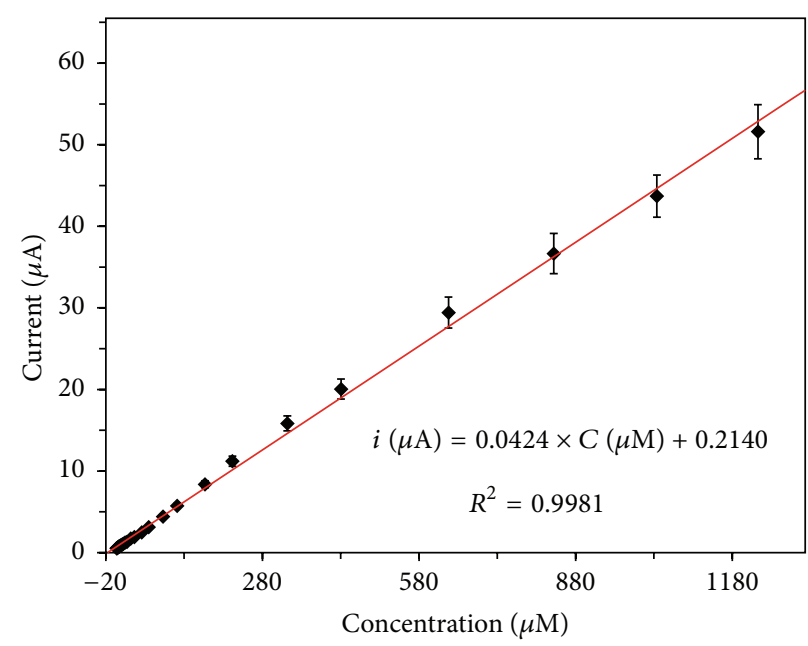

(b)

Figure 7: (a) Typical amperometric response of the CNT-PdPt electrode in 0.1 M PBS buffer solution (pH 7.4) during successive addition of different concentrations $(0.55-200 \mu \mathrm{M})$ of hydrazine $(\mathrm{A} \rightarrow \mathrm{B}, 0.55 \mu \mathrm{M} ; \mathrm{B} \rightarrow \mathrm{C}, 0.95 \mu \mathrm{M} ; \mathrm{C} \rightarrow \mathrm{D}, 1.8 \mu \mathrm{M} ; \mathrm{D} \rightarrow \mathrm{E}, 3.5 \mu \mathrm{M} ; \mathrm{E} \rightarrow \mathrm{F}, 7.0 \mu \mathrm{M} ; \mathrm{F}$ $\rightarrow \mathrm{G}, 13.8 \mu \mathrm{M} ; \mathrm{G} \rightarrow \mathrm{H}, 27 \mu \mathrm{M} ; \mathrm{H} \rightarrow \mathrm{I}, 53 \mu \mathrm{M} ; \mathrm{I} \rightarrow \mathrm{J}, 103.6 \mu \mathrm{M} ; \mathrm{J} \rightarrow 200 \mu \mathrm{M})$. The applied potential was $0.2 \mathrm{~V}$ versus SCE. Inset is the enlarged view of the amperometric response up to $1.8 \mu \mathrm{M}$ hydrazine. (b) Current-concentration calibration graph exhibiting a linear relationship with the steady state current and hydrazine concentration. Error bars are the standard error of the mean ( $n=5$ electrodes).
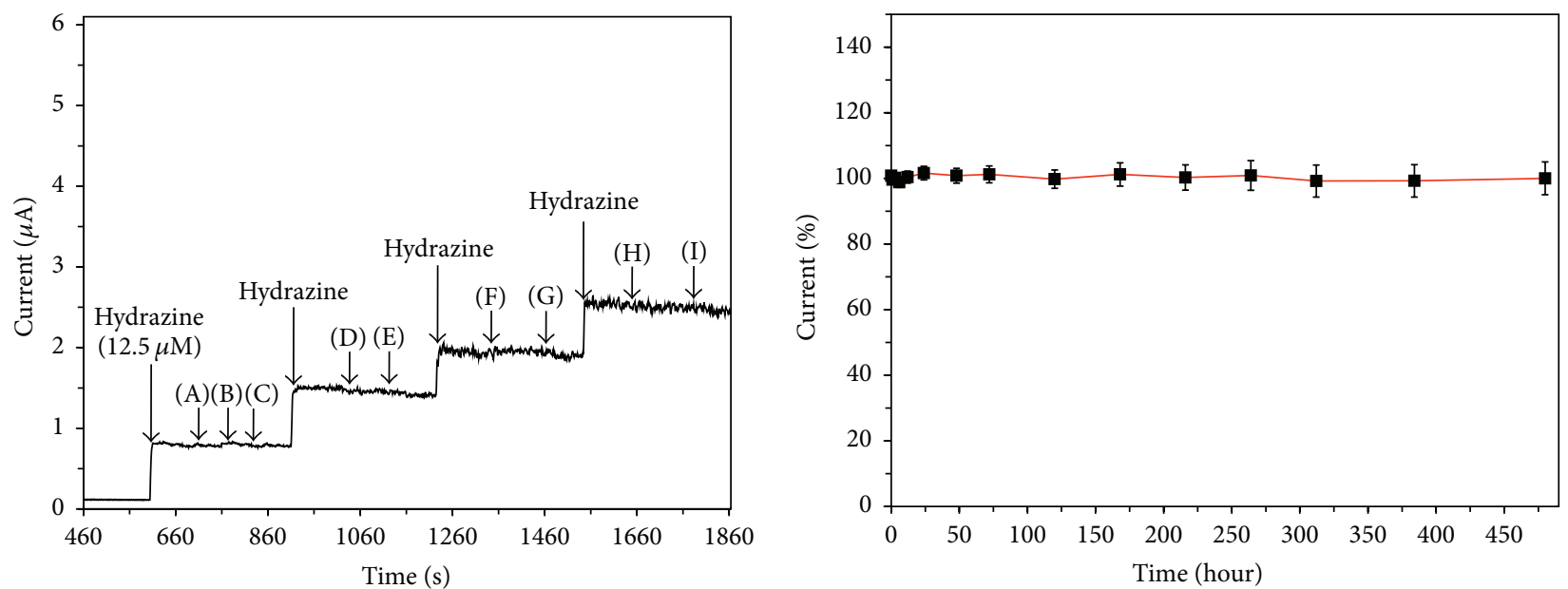
(A) $\mathrm{NH}_{3}$
(F) $\mathrm{Cl}^{-}$
(B) $\mathrm{NO}_{2}^{-}$
(G) $\mathrm{SO}_{4}{ }^{2-}$
(C) $\mathrm{NO}_{3}{ }^{-}$
(H) Ethanol
(D) $\mathrm{Na}^{+}$
(I) Glucose
(E) $\mathrm{Ca}^{2+}$

(a)

(b)

Figure 8: (a) Amperometric response of the CNT-PdPt electrode after successive addition of $12.5 \mu \mathrm{M}$ hydrazine and 8-fold concentration of different interfering species. Supporting electrolyte: 0.1 M PBS (pH 7.4). Applied potential: 0.2 V. (b) Changes in current (\%) of the CNT-PdPt monitored in $0.1 \mathrm{M}$ PBS ( $\mathrm{pH}$ 7.4) containing $25 \mu \mathrm{M}$ hydrazine for 15 repetitive measurements during 20 days. The initial response was set as $100 \%$. Error bars are the standard error of the mean ( $n=5$ electrodes).

selectivity for hydrazine detection. The reproducibility of CNT-PdPt was verified by measuring the oxidation currents in five different solutions in the presence of $25 \mu \mathrm{M}$ hydrazine with a freshly prepared electrode for each determination. A relative standard deviation (RSD) of $1.1 \%$ was observed, which indicates excellent reproducibility. The reusability and storage stability of the electrode were studied by using the same modified electrode in 15 repeated exposures to $25 \mu \mathrm{M}$ hydrazine. After each experiment, the electrode was washed with PBS solution, dried at room temperature, and stored 
TABLE 2: Determination of hydrazine in tap water samples spiked with different amounts of hydrazine $(n=5)$.

\begin{tabular}{ccccc}
\hline Real sample Added $(\mu \mathrm{M})$ & Found $(\mu \mathrm{M})$ & Recovery $(\%)$ & RSD (\%) \\
\hline \multirow{4}{*}{ Tap water } & 20.0 & 21.2 & 106.0 & 3.26 \\
& 60.0 & 62.3 & 103.8 & 1.97 \\
& 80.0 & 81.7 & 102.1 & 1.54 \\
& 100.0 & 102.4 & 102.4 & 1.76 \\
\hline
\end{tabular}

in air at room temperature. As shown in Figure 8(b), the electrode retained $99 \%$ of its initial current after 20 days of repeated use with exposure to air and no noticeable potential shift was observed. This result indicates excellent reusability and storage stability. The sensor's advantages of simple preparation, good linear response range, reusability, and storage stability mean that CNT-PdPt is a promising electron mediator for the fabrication of efficient amperometric sensors for the detection of hydrazine.

Finally, tap water was used in real sample analysis to evaluate the applicability of the proposed sensor. Since no hydrazine was detected in the samples, the water samples were spiked with different standard concentrations of hydrazine. The samples were analyzed by amperometric method after adjusting $\mathrm{pH}$ to 7.4 , using $0.1 \mathrm{M}$ PBS. The obtained results are shown in Table 2 . The recoveries for the determination of hydrazine were from 102.1 to $106.0 \%$ and the RSD was below $4 \%(n=5)$, indicating that the CNTPdPt nanocomposites should be a promising sensor for the effective determination of hydrazine.

\section{Conclusions}

In summary, PdPt bimetallic nanoparticles were synthesized with the simple chemical reduction method. The nanocomposites obtained by depositing PdPt as-prepared nanoparticles on thiolated CNT surfaces were used as efficient electron mediators for the fabrication of amperometric hydrazine sensors. The atomic \% of the Pd and Pt contained within the CNT-PdPt nanocomposites was found to be nearly 3.0 and $3.2 \%$, respectively. The amperometric responses reached steady state signals within 5 seconds. The obtained linear response range and detection limit for the CNT-PdPt sensor were found to be $0.55-1,200 \mu \mathrm{M}$ and $0.28 \mu \mathrm{M}$, respectively. The obtained detection limit and linear range are comparable to or better than the results for previously reported chemically modified electrodes for the determination of hydrazine. Moreover, the CNT-PdPt sensor was found to exhibit good anti-interference ability, reusability, and long-term storage stability. This strategy of combining bimetallic nanoparticles and thiolated CNTs has been shown to have significant advantages for the fabrication of reusable hydrazine sensors with a fast response time and a low detection limit.

\section{Conflict of Interests}

The authors declare that there is no conflict of interests regarding the publication of this paper.

\section{Acknowledgments}

This study was financially supported by Chonnam National University, 2015. The authors are thankful for TEM and Raman measurements from the Korea Basic Science Institute (KBSI), Gwangju branch in Chonnam National University.

\section{References}

[1] A. Umar, M. M. Rahman, S. H. Kim, and Y.-B. Hahn, "Zinc oxide nanonail based chemical sensor for hydrazine detection," Chemical Communications, no. 2, pp. 166-168, 2008.

[2] S. Garrod, M. E. Bollard, A. W. Nicholls et al., "Integrated metabonomic analysis of the multiorgan effects of hydrazine toxicity in the rat," Chemical Research in Toxicology, vol. 18, no. 2, pp. 115-122, 2005.

[3] E. H. Vernot, J. D. MacEwen, R. H. Bruner et al., "Long-term inhalation toxicity of hydrazine," Fundamental and Applied Toxicology, vol. 5, no. 6, pp. 1050-1064, 1985.

[4] A. Poso, A. V. Wright, and J. Gynther, "An empirical and theoretical study on mechanisms of mutagenic activity of hydrazine compounds," Mutation Research, vol. 332, no. 1-2, pp. 63-71, 1995.

[5] M. A. Kamyabi, O. Narimani, and H. H. Monfared, "Electrocatalytic oxidation of hydrazine using glassy carbon electrode modified with carbon nanotube and terpyridine manganese(II) complex," Journal of Electroanalytical Chemistry, vol. 644, no. 1, pp. 67-73, 2010.

[6] J. Li, H. Xie, and L. Chen, "A sensitive hydrazine electrochemical sensor based on electrodeposition of gold nanoparticles on choline film modified glassy carbon electrode," Sensors and Actuators B: Chemical, vol. 153, no. 1, pp. 239-245, 2011.

[7] H. M. Nassef, A.-E. Radi, and C. K. O’Sullivan, "Electrocatalytic oxidation of hydrazine at o-aminophenol grafted modified glassy carbon electrode: reusable hydrazine amperometric sensor," Journal of Electroanalytical Chemistry, vol. 592, no. 2, pp. 139-146, 2006.

[8] L. Wang, C. Hu, Y. Nemoto, Y. Tateyama, and Y. Yamauchi, "On the role of ascorbic acid in the synthesis of single-crystal hyperbranched platinum nanostructures," Crystal Growth and Design, vol. 10, no. 8, pp. 3454-3460, 2010.

[9] C. Li and Y. Yamauchi, "Facile solution synthesis of Ag@Pt coreshell nanoparticles with dendritic Pt shells," Physical Chemistry Chemical Physics, vol. 15, no. 10, pp. 3490-3496, 2013.

[10] H. Ataee-Esfahani, M. Imura, and Y. Yamauchi, "All-metal mesoporous nanocolloids: solution-phase synthesis of coreshell Pd@Pt nanoparticles with a designed concave surface," Angewandte Chemie -International Edition, vol. 52, no. 51, pp. 13611-13615, 2013.

[11] A. P. O’Mullane, S. E. Dale, J. V. Macpherson, and P. R. Unwin, "Fabrication and electrocatalytic properties of polyaniline $/ \mathrm{Pt}$ nanoparticle composites," Chemical Communications, vol. 10, no. 14, pp. 1606-1607, 2004.

[12] N. Maleki, A. Safavi, E. Farjami, and F. Tajabadi, "Palladium nanoparticle decorated carbon ionic liquid electrode for highly efficient electrocatalytic oxidation and determination of hydrazine," Analytica Chimica Acta, vol. 611, no. 2, pp. 151-155, 2008.

[13] Y. Shen, Q. Xu, H. Gao, and N. Zhu, "Dendrimer-encapsulated Pd nanoparticles anchored on carbon nanotubes for electrocatalytic hydrazine oxidation," Electrochemistry Communications, vol. 11, no. 6, pp. 1329-1332, 2009. 
[14] M. Shamsipur, Z. Karimi, M. A. Tabrizi, and A. Shamsipur, "Electrocatalytic determination of traces of hydrazine by a glassy carbon electrode modified with palladium-gold nanoparticles," Electroanalysis, vol. 26, no. 9, pp. 1994-2001, 2014.

[15] C. Karuppiah, S. Palanisamy, S.-M. Chen, S. K. Ramaraj, and P. Periakaruppan, "A novel and sensitive amperometric hydrazine sensor based on gold nanoparticles decorated graphite nanosheets modified screen printed carbon electrode," Electrochimica Acta, vol. 139, pp. 157-164, 2014.

[16] R. Devasenathipathy, V. Mani, S.-M. Chen, D. Arulraj, and V. S. Vasantha, "Highly stable and sensitive amperometric sensor for the determination of trace level hydrazine at cross linked pectin stabilized gold nanoparticles decorated graphene nanosheets," Electrochimica Acta, vol. 135, pp. 260-269, 2014.

[17] G. Gao, D. Guo, C. Wang, and H. Li, "Electrocrystallized Ag nanoparticle on functional multi-walled carbon nanotube surfaces for hydrazine oxidation," Electrochemistry Communications, vol. 9, no. 7, pp. 1582-1586, 2007.

[18] A. P. O’Mullane, S. E. Dale, T. M. Day, N. R. Wilson, J. V. MacPherson, and P. R. Unwin, "Formation of polyaniline/Pt nanoparticle composite films and their electrocatalytic properties," Journal of Solid State Electrochemistry, vol. 10, no. 10, pp. 792-807, 2006.

[19] A. Krittayavathananon, P. Srimuk, S. Luanwuthi, and M. Sawangphruk, "Palladium nanoparticles decorated on reduced graphene oxide rotating disk electrodes toward ultrasensitive hydrazine detection: effects of particle size and hydrodynamic diffusion," Analytical Chemistry, vol. 86, no. 24, pp. 12272-12278, 2014.

[20] B. J. Gallon, R. W. Kojima, R. B. Kaner, and P. L. Diaconescu, "Palladium nanoparticles supported on polyaniline nanofibers as a semi-heterogeneous catalyst in water," Angewandte Chemie-International Edition, vol. 46, no. 38, pp. 72517254, 2007.

[21] W.-J. Liu, T.-T. Qian, and H. Jiang, "Bimetallic Fe nanoparticles: recent advances in synthesis and application in catalytic elimination of environmental pollutants," Chemical Engineering Journal, vol. 236, pp. 448-463, 2014.

[22] L. A. Zook and J. Leddy, "Density and solubility of nafion: recast, annealed, and commercial films," Analytical Chemistry, vol. 68, no. 21, pp. 3793-3796, 1996.

[23] F. Alcaide, G. Álvarez, P. L. Cabot, O. Miguel, and A. Querejeta, "Performance of carbon-supported PtPd as catalyst for hydrogen oxidation in the anodes of proton exchange membrane fuel cells," International Journal of Hydrogen Energy, vol. 35, no. 20, pp. 11634-11641, 2010.

[24] G. Zhang, Z.-G. Shao, W. Lu, F. Xie, X. Qin, and B. Yi, “Electrochemical preparation and characterization of PdPt nanocages with improved electrocatalytic activity toward oxygen reduction reaction," Electrochimica Acta, vol. 103, pp. 66-76, 2013.

[25] B. Lim, M. Jiang, P. H. C. Camargo et al., "Pd-Pt bimetallic nanodendrites with high activity for oxygen reduction," Science, vol. 324, no. 5932, pp. 1302-1305, 2009.

[26] Y. Li, X. Fan, J. Qi et al., "Gold nanoparticles-graphene hybrids as active catalysts for Suzuki reaction," Materials Research Bulletin, vol. 45, no. 10, pp. 1413-1418, 2010.

[27] Y.-K. Kim, H.-K. Na, and D.-H. Min, "Influence of surface functionalization on the growth of gold nanostructures on graphene Thin Films," Langmuir, vol. 26, no. 16, pp. 1306513070, 2010.

[28] C. L. Hedberg, Handbook of Auger Electron Spectroscopy, Physical Electronics, Chanhassen, Minn, USA, 3rd edition, 1995.
[29] M. M. Ardakani, P. E. karami, P. Rahimi, H. R. Zare, and H. Naeimi, "Electrocatalytic hydrazine oxidation on quinizarine modified glassy carbon electrode," Electrochimica Acta, vol. 52, no. 20, pp. 6118-6124, 2007.

[30] H. Ahmar, S. Keshipour, H. Hosseini, A. R. Fakhari, A. Shaabani, and A. Bagheri, "Electrocatalytic oxidation of hydrazine at glassy carbon electrode modified with ethylenediamine cellulose immobilized palladium nanoparticles," Journal of Electroanalytical Chemistry, vol. 690, pp. 96-103, 2013.

[31] M. Xin, H. Lin, J. Yang, M. Chen, X. Ma, and J. Liu, "Preparation of polyaniline/ $\mathrm{Au}_{0}$ nanocomposites modified electrode and application for hydrazine detection," Electroanalysis, vol. 26, no. 10, pp. 2216-2223, 2014.

[32] A. J. Bard and L. R. Faulkner, Electrochemical Methods, Fundamentals and Applications, Wiley, New York, NY, USA, 1980.

[33] S. Antoniadou, A. D. Jannakoudakis, and E. Theodoridou, "Electrocatalytic reactions on carbon fibre electrodes modified by hemine II. Electro-oxidation of hydrazine," Synthetic Metals, vol. 30, no. 3, pp. 295-304, 1989.

[34] B. Zargar and A. Hatamie, "A simple and fast colorimetric method for detection of hydrazine in water samples based on formation of gold nanoparticles as a colorimetric probe," Sensors and Actuators B: Chemical, vol. 182, pp. 706-710, 2013.

[35] M. Rajkumar, C. P. Hong, and S.-M. Chen, "Electrochemical synthesis of palladium nano urchins decorated multi walled carbon nanotubes for electrocatalytic oxidation of hydrazine and reduction of hydrogen peroxide," International Journal of Electrochemical Science, vol. 8, no. 4, pp. 5262-5274, 2013.

[36] L. Shang, F. Zhao, and B. Zeng, "Electrocatalytic oxidation and determination of hydrazine at an $\mathrm{AuCu}$ nanoparticlesgraphene-ionic liquid composite film coated glassy carbon electrode," Electroanalysis, vol. 24, no. 12, pp. 2380-2386, 2012.

[37] X. Qin, Q. Li, A. M. Asiri, A. O. Al-Youbi, and X. Sun, "One-pot synthesis of $\mathrm{Au}$ nanoparticles/reduced graphene oxide nanocomposites and their application for electrochemical $\mathrm{H}_{2} \mathrm{O}_{2}$, glucose, and hydrazine sensing," Gold Bulletin, vol. 47, no. 1-2, pp. 3-8, 2014.

[38] H. Lin, J. Yang, J. Liu, Y. Huang, J. Xiao, and X. Zhang, "Properties of Pd nanoparticles-embedded polyaniline multilayer film and its electrocatalytic activity for hydrazine oxidation," Electrochimica Acta, vol. 90, pp. 382-392, 2013. 

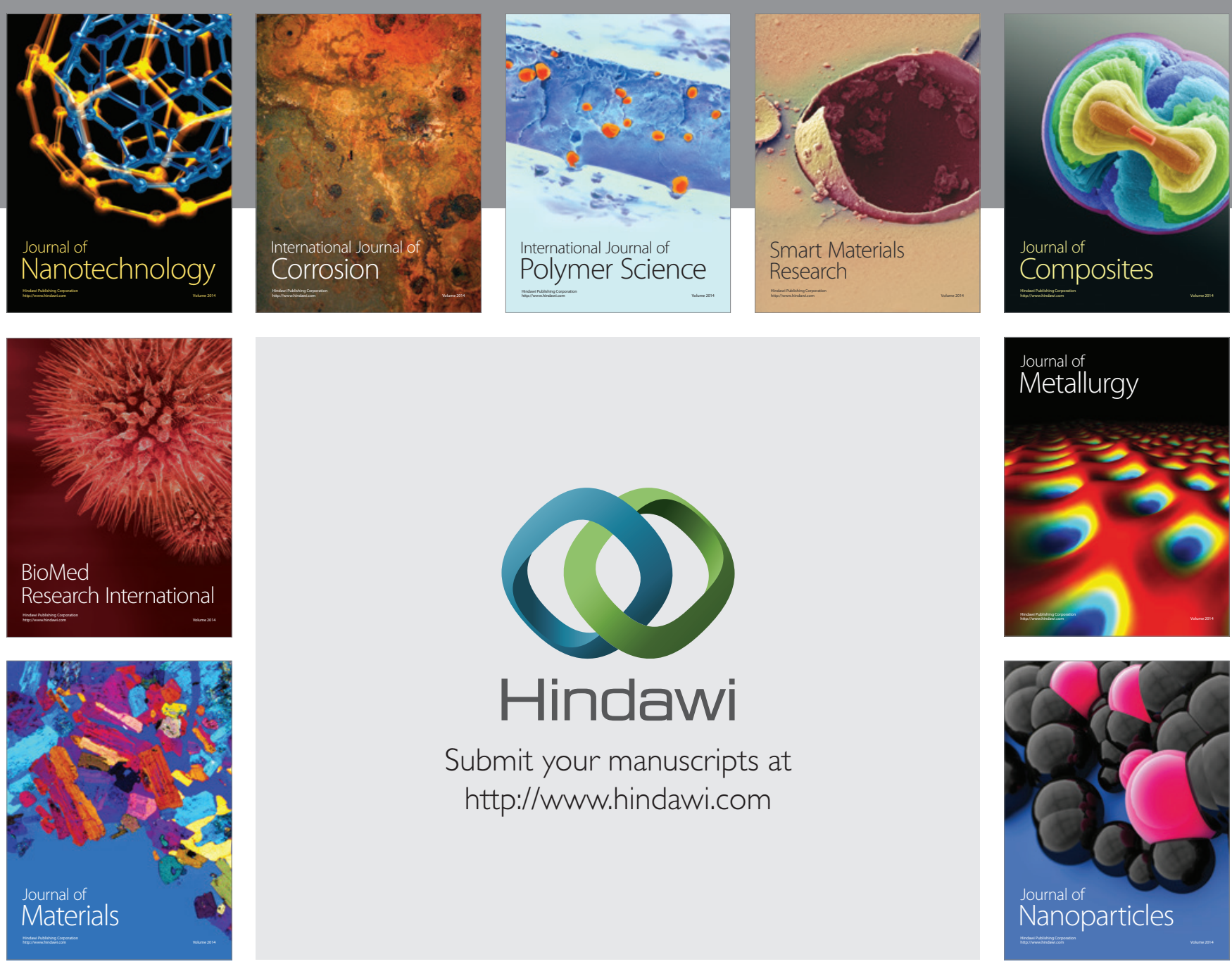

Submit your manuscripts at http://www.hindawi.com
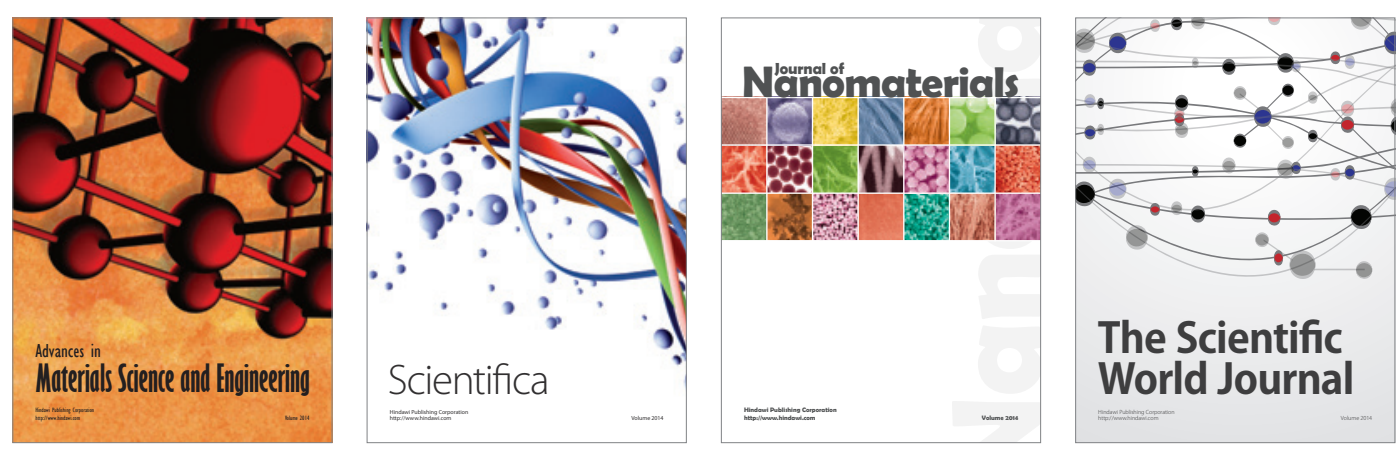

\section{The Scientific World Journal}
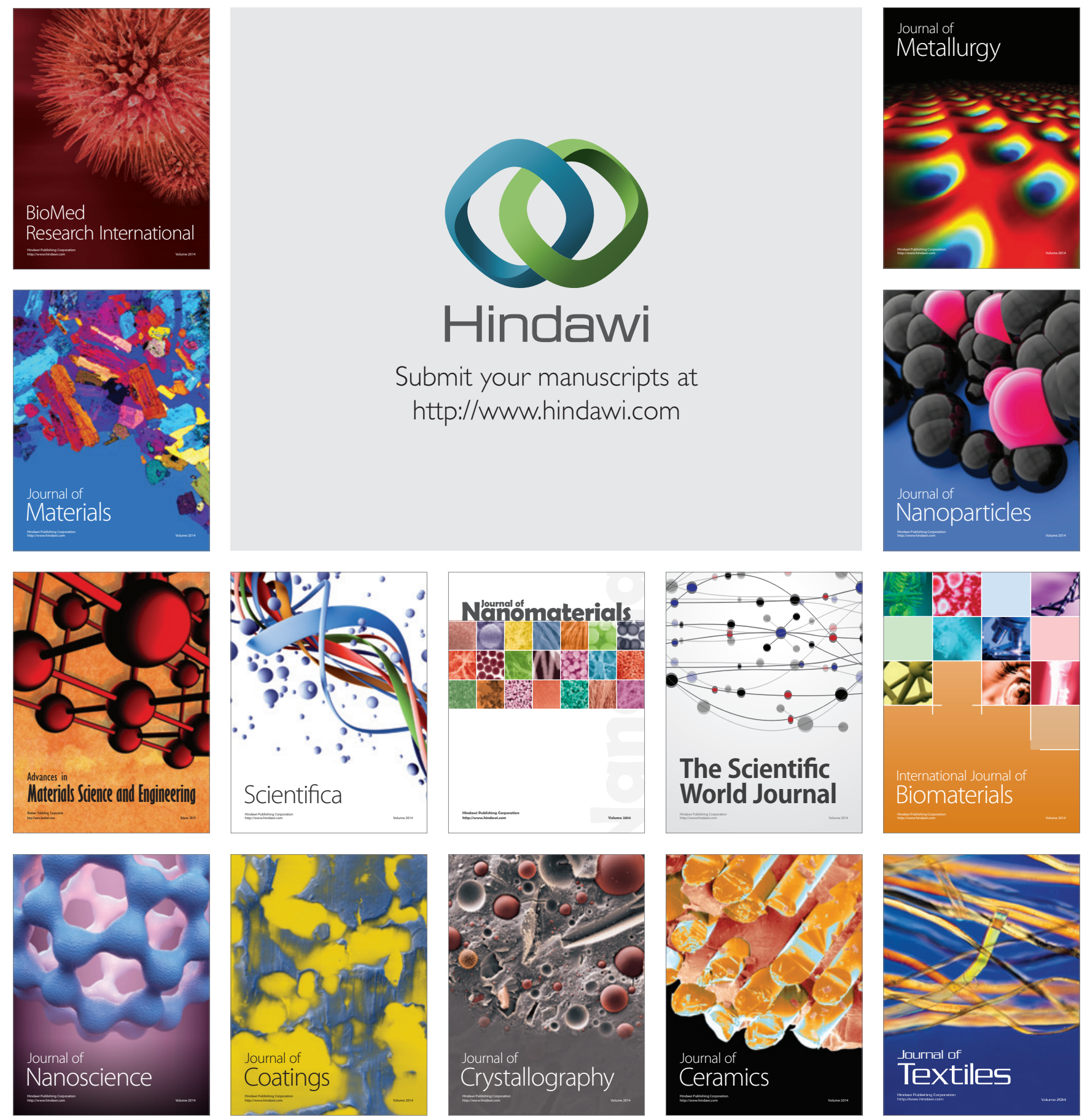